\title{
Evolution of Advanced Clustering Methods in WSN: A Review
}

\author{
Neeti Jain \\ M.Tech Student \\ Department of Computer Science \\ Government Engineering College Ajmer, Rajasthan, \\ India
}

\author{
Prakriti Trivedi \\ Asst Professor \\ Department of Computer Science \\ Government Engineering College Ajmer, Rajasthan, \\ India
}

\begin{abstract}
The use of wireless sensor networks has grown tremendously in the last decade, wireless sensor networks are used in wide variety of applications. Nodes in WSN have limited battery lifetime that affects the performance of the network. Scalability is also a major concern. The use of clustering in WSN provides a solution to this. Many improvements have been made in the basic clustering protocols .In this paper, we will review the most recent proposals and implementations of the clustering algorithms used in WSN. We will also try to figure out pros and cons of their working. In the last we will compare the performances of various algorithms.
\end{abstract}

\section{Keywords}

Wireless sensor networks, clustering methods, cluster head, base station, energy balancing

\section{INTRODUCTION}

With the advances in the MEMS technology there has been recorded a considerable growth in wireless technology. Wireless sensor networks find its applications in a wide variety of areas such as environmental monitoring, forest fire detection, military applications, target tracking etc [1]. Wireless sensor networks consist of a large collections of nodes deployed either randomly or manually in a particular area of interest.

WSN have its own unique characteristics when compared with mobile ad- hoc network (MANET), such as the sensor nodes have limited memory and computational capacities, there are a much larger number of nodes used than in MANET, the network is scalable, nodes are densely deployed, means of communication is generally a broadcast mechanism rather point to point as in MANET, the application is data centric. These characteristics poses new design challenges to the research community and continues work is been done in this field.

The sensor nodes have four main components a sensing unit, a processing unit, a transceiver unit and a power unit. Depending on the application there can be additional components such as location finding system, a power generator and a mobilizer [1]. Senor nodes communicate with each other either directly or through intermediate nodes for collecting and transferring information to the base station (BS). The location of BS is generally fixed and it is located far away from the nodes. As the sensor nodes have limited transmission range they can use multi hop communication i.e. sensing the collected data thorough intermediate nodes which saves the energy of the node sending data. The topology of the sensor network keeps changing over time.
Due to various constraints such as limited power, maximizing lifetime, balancing energy consumption within the network need of data aggregation arises so that transmission of redundant messages within the network can be avoided. The technique commonly used for data aggregation in WSN is clustering i.e. grouping the nodes into small manageable groups. Clustering also helps to meet the objectives of scalability in the network.

Each cluster has a cluster head $(\mathrm{CH})$ the members of the cluster send the sensed data to the $\mathrm{CH}$ which then aggregates the collected data for transmitting it to the BS. The $\mathrm{CH}$ can directly transmit the data to the BS or can use intermediate $\mathrm{CH}$ 's. The election of $\mathrm{CH}$ may depend on several factors such as its residual energy, distance from the BS etc. Since the $\mathrm{CH}$ performs the main task of data aggregation and also act as intermediate node for other CH's it may drain its energy earlier then the other members of the cluster, so the $\mathrm{CH}$ is reelected after each round.

In this paper we will review working of the clustering algorithms used in WSN and their improvements, we will study their advantages and disadvantages and their effect on network performance. The paper is organized as: in section II basic clustering algorithms are presented, in section III improvements made $\mathrm{n}$ the basic clustering protocols are studied, Section IV describes architecture based clustering protocols, Section V describes sectoring based clustering protocols, section VI contains results and section VII is conclusion.

\section{Basic Clustering Protocols in WSN}

Linked Cluster Algorithm (LCA) [2] - Among the earliest work on clustering was proposed by Baker \& Ephremides, the focus in on constructing a network topology that can handle mobility of nodes in the network. By clustering, cluster heads $(\mathrm{CHs})$ are hoped to form a backbone network to which cluster members can connect while they are moving. The main goal of this proposed distributed algorithm is to form clusters so that a $\mathrm{CH}$ is directly connected to all nodes that are in its clusters. The LCA algorithm helps in maximizing network connectivity to a great extent.

A node is assigned the slots in the form of frame; the frames should match the ID of the nodes. In the first round each node broadcast it ID and listens to transmissions of other nodes. In the next round, a node broadcast the neighbor IDs that it has heard in the first round and so each node knows to which other node it is bidirectional linked, and consequently each node knows its 1-hop and 2-hop neighbors. A node ' $\mathrm{x}$ ' is assigned to become a $\mathrm{CH}$ if it has the highest ID among its neighbors or if it does not have the highest ID in its 1-hop 
neighborhood, but if there exists at least one neighboring node ' $y$ ' such that ' $x$ ' is the highest ID node in $y$ 's 1-hop neighborhood. The LCA algorithm results into a large number of clusters so this approach is modified.

Hierarchical control clustering [3]-The goal of scheme proposed by Banerjee and Khuller was to form a multi-tier hierarchical clustering. Various cluster properties such as cluster size and the degree of overlap are considered so that scalability in hierarchy is maintained. In the proposed algorithm any node in the network can start the cluster formation process. If many nodes start the cluster formation process at the same time them the preference is given to the node having the least node ID. The algorithm is broadly divided into two phases: Tree discovery phase and the cluster formation phase.

The tree discovery phase basically consists of the formation of a Breadth First Search Tree that is rooted at the initiator node. Each node ' $x$ ' broadcasts a signal once every ' $p$ ' units of time carrying the information about its shortest hop-distance to the root, ' $r$ '. A node ' $y$ ' that is neighbor of ' $x$ ' will choose ' $x$ ' to be its parent and will update its hop-distance to the root, if the route through ' $\mathrm{x}$ ' is shorter. The signal that is broadcasted carry the information such as source ID, parent ID, root ID, and sub-tree size. Every node updates the size of its sub-tree when the size of its children sub-trees changes.

The cluster formation phase is imitated when a sub-tree of a node crosses the size parameter ' $\mathrm{k}$ '. The node imitates cluster formation process on its sub-tree. A single cluster is formed single for the entire sub-tree if size of sub-tree is $<2 \mathrm{k}$, else, multiple clusters are formed. After the clusters are created, keeping cluster information is crucial task for the clusters while maintaining the BFS tree is unimportant. This approach can handle dynamic environment.

\section{Low Energy Adaptive Clustering Hierarchy} (LEACH) [4] - LEACH is among the most popular clustering algorithm for WSN. It is a hierarchical, distributed, probabilistic protocol that uses one hop routing. The main objective is to maximize the network lifetime by evenly distributing the energy consumption among all nodes of the network. The clusters are formed based on the received signal strength and the $\mathrm{CH}$ nodes are used as routers to the basestation. The entire data processing task such as data fusion and aggregation are locally performed by the $\mathrm{CH}$. A distributed algorithm is used for cluster formation in LEACH, where the nodes make autonomous decisions without any centralized control. A node has a probability $p$ for becoming a $\mathrm{CH}$ and after it decides it broadcasts its decision. The nodes which are not $\mathrm{CHs}$ select their clusters by choosing the $\mathrm{CH}$ that can be reached using the least communication energy. The role of being a $\mathrm{CH}$ is periodically rotated among the nodes of the cluster in order to balance the energy load. To perform rotation each node chooses a random number between 0 and 1. A node becomes a $\mathrm{CH}$ for the current round rotation if the number is less than the threshold:

$$
T(i)=\left\{\begin{aligned}
\frac{p}{1-p * \operatorname{rmod} \frac{1}{p}}, & \text { ifi } \epsilon G \\
0, & \text { otherwise }
\end{aligned}\right.
$$

Where $p$ is the desired percentage of $\mathrm{CH}$ nodes, $r$ is the current round number, and $G$ is the set of nodes that have not been $\mathrm{CHs}$ in the last $\frac{1}{p}$ rounds.
Generally, LEACH can provide a uniform load distribution in one-hop sensor networks. Moreover, it also provides a good balancing of energy consumption by randomly rotating $\mathrm{CHs}$. Furthermore, the localized coordination scheme is used in LEACH that provides better scalability for cluster formation, whereas the better load balancing enhances the network lifetime.

There are some drawbacks of the LEACH algorithm, as A probabilistic approach is used to elect $\mathrm{CHs}$ so there are chances that a low energy node gets selected as $\mathrm{CH}$. When this node dies, the whole cell becomes dysfunctional. This can also lead to chances that the $\mathrm{CH}$ election is concentrated to one part of the network and some nodes do not have any $\mathrm{CH}$ in their range Also it is assumed that $\mathrm{CHs}$ have long communication range so that data can be directly transmitted to the base station, but this approach is not realistic since the base station is often not directly reachable. In LEAH one hop intra and inter cluster topology is used wherein each node can directly transmit data to $\mathrm{CH}$ and the $\mathrm{CH}$ to the base station.

\section{Hybrid Energy-Efficient Distributed Clustering} (HEED) [5]-It is a distributed, hierarchical clustering algorithm wherein single hop communication is used within the clusters and multi-hop communication is used between $\mathrm{CH}$ and BS. While selecting $\mathrm{CH}$ nodes two parameters are taken into account residual energy and intra cluster communication cost. While probably choosing the initial set of $\mathrm{CHs}$ residual energy is used. The intra cluster communication cost helps a node in deciding whether to join a cluster or not. There are some important characteristics of the HEED algorithm.

- Unlike LEACH the CH are not selected randomly, only nodes with high residual energy can become $\mathrm{CHs}$

- There is a low probability of two nodes that are within the transmission range of each other becoming $\mathrm{CH}$; this means that unlike LEACH the $\mathrm{CH}$ nodes are well distributed in the network.

- A node when choosing a cluster, will communicate with that $\mathrm{CH}$ that results into lowest communication cost. In HEED each node can only be a part of one cluster and can communicate directly to the $\mathrm{CH}$ using one hop routing.

\section{Improvements in the Basic Clustering Protocols}

Energy-efficient hierarchical clustering (EEHC) [6] -Bandyopadhyay and Coyle proposed EEHC; a distributed, randomized clustering algorithm for WSNs with the main goal of maximizing network lifetime. The EEHC algorithm organizes the sensors in the network into clusters with a hierarchy of $\mathrm{CHs}$. Each $\mathrm{CH}$ collects data from its cluster members and sends an aggregated report through a hierarchy of $\mathrm{CHs}$ to the Base Station. The assumption for the EEHC algorithm is that the communication environment is error free.

The algorithm proceeds in two stages; initial and extended. In the initial stage each node becomes a $\mathrm{CH}$ with a probability $p$ and announces itself as the $\mathrm{CH}$ to nodes within its communication range. These cluster head are termed as volunteer CHs. Nodes that receives such an announcement and are not CHS itself joins the cluster that is closest to them. Nodes which are not a part of any cluster and that are neither 
CH's, itself become CHs. These CHs are called forced CHs. If the announcement does not reach to a node within a preset time interval $t$ that is calculated based on the duration for a packet to reach a node that is $\mathrm{k}$ hops away, the node will become a forced $\mathrm{CH}$ assuming that it is not within $\mathrm{k}$ hops of all volunteer $\mathrm{CHs}$

Now the process is extended in the second stage of the algorithm to perform multi-level clustering, i.e. building $h$ levels of cluster hierarchy. The algorithm ensures that there is $\mathrm{h}$ hop connectivity between $\mathrm{CHs}$ and base station. Level $\mathrm{h}$ is assumed to be the highest level and the sensor nodes transmit the aggregated data to the lowest level i.e. level- 1 . The $\mathrm{CHs}$ at the lowest level i.e. Level-1 transmits the aggregated data to the level-2 $\mathrm{CHs}$ and so on. The $\mathrm{CH}$ at the top level of the clustering hierarchy transmits the aggregated data report to the base station. The energy consumption of the network depends on the $\mathrm{p}$ and $\mathrm{k}$ parameters of the algorithm. The time complexity of EEHC algorithm is $\mathrm{O}\left(\mathrm{k}_{1}+\mathrm{k}_{2}+\ldots+\mathrm{k}_{\mathrm{h}}\right)$, which is a significant improvement over the many clustering algorithms having $\mathrm{O}(\mathrm{n})$ time complexity.

ACTH-LEACH [7]-In this paper a modification of LEACH algorithm is proposed by using two hop transmissions. The two hop LEACH protocol (ACTHLEACH) proposed improves network lifetime, an adaptive algorithm is used for multi hop transmission and cluster head election. According to distance from BS nodes are tagged as near or far, all the near nodes belong to one cluster while the far nodes use the Greedy $\mathrm{K}$ means algorithm for division into different clusters. To balance the energy load the node having maximum residual energy is selected as the $\mathrm{CH}$. To communicate with $\mathrm{BS}$ far $\mathrm{CH}$ uses near $\mathrm{CH}$, if it is alive else direct communication is used.

The ACTH-LEACH protocol follows three steps clustering, cluster head election, data transmission. In the clustering phase based on a threshold $d_{o}$ nodes are tagged as near and far, if a node distance is less than $d_{o}$ it is tagged as near. Greedy $\mathrm{K}$ - means algorithm is used for formation of clusters in the far area .In the cluster head selection step after an initial $\mathrm{CH}$ is selected, based on residual energy $\mathrm{CH}$ is rotated to balance energy load. The node having maximum residual energy is selected as $\mathrm{CH}$. This saves energy of all the nodes since broadcasting information within their own clustering area is required now, other than $\mathrm{LEACH}$ where $\mathrm{CH}$ broadcasts for the entire network. In the two hop transmission phase the far $\mathrm{CH}$ send data to near $\mathrm{CH}$ if it is alive i.e. two hop transmissions is used else far $\mathrm{CH}$ uses direct one hop transmission.

The ACTH-LEACH is an improvement on basic LEACH algorithm, it prolongs network lifetime and is more energy efficient. The algorithm can be improved by optimizing the threshold value.

$T L-L E A C H$ [8]-It is an improvement in the basic LEACH algorithm. The algorithm proceeds in three phases: $\mathrm{CH}$ election, formation of clusters and data transmission. Each cluster has two CH's namely primary and secondary, the secondary $\mathrm{CH}$ communicates with other members of the cluster and aggregates the data which is then transmitted to the primary $\mathrm{CH}$. The primary $\mathrm{CH}$ transmits the data to base station. the data send to base station is reduced considerably by the algorithm but there is a lot of overhead involved in selecting the primary and the secondary $\mathrm{CH}^{\prime}$ 's which consumes a lot of energy thereby affecting the lifetime of the network, also the algorithm is not energy efficient.

HCTE [9]-HCTE is clustering based routing algorithm that takes into account balancing of energy between nodes as its main design concern. In the algorithm there are two cluster heads in each cluster namely initial and second cluster head. HCTE uses multi hop routing for transmitting data from $\mathrm{CH}$ to sink. The algorithm proceeds in five phases: the first step is initial $\mathrm{CH}$ announcement in which the probability of nodes becoming $\mathrm{CH}$ is calculated .In second step cluster formation takes place; nodes join the clusters based on their confidence value or depending on their transmission range. In the third step the second $\mathrm{CH}$ is announced based on confidence value, the node having highest confidence value among cluster members is elected as second $\mathrm{CH}$. The schedule creation phase follows the same procedure as that of LEACH. In last step data transmission takes place.

\section{Architecture based Clustering Protocols.} Distributed Weight-Based Energy-Efficient Hierarchical Clustering (DWEHC) [10] -It is a distributed algorithm which aims at high energy efficiency by generating balanced cluster sizes and optimizing the intracluster topology. DWEHC has O (1) time complexity. Each sensor calculates its weight after locating the neighboring nodes in its area. Sensors residual energy and its distance to neighboring nodes together decides the weight function, the node having largest weight is elected as $\mathrm{CH}$. At this point the nodes are considered as first level members because they are directly linked to the $\mathrm{CH}$. A node progressively adjusts such membership in order to reach a $\mathrm{CH}$ using the least amount of energy. A node checks with its neighbors which are non $\mathrm{CH}$ to find out their minimal cost of reaching a $\mathrm{CH}$. Once the node has knowledge of the distance to its neighbors, it can analyze whether it is better to stay a first-level member or become a second-level one i.e. reaching the $\mathrm{CH}$ in two -hops. The process continues until nodes settles on the most energy efficient intra-cluster topology. To minimize the number of levels, every cluster is assigned a range within which member nodes should lay.

DWEHC and HEED have many similarities: every node in the network participates in clustering, no assumptions are made about the network size and residual energy is considered in $\mathrm{CH}$ selection. Despite of having such similarities there are many differences in terms of performance. The clusters generated by DWEHC are more balanced than HEED also DWEHC have lower energy consumption than HEED in intra and inter cluster communication.

EECS [11] - in EECS the focus of the problem is on the fact that clusters that are at a greater distance from the base station requires more energy for data transmission than the once that are near to it. For this dynamic sizing of the clusters is done which is based on cluster distance from the base station. The algorithm provides equal distribution of energy prolonging network lifetime also full connectivity can be provided for a longer duration of time.

\section{A New Approach Layered Architecture based} Clustering for Prolong Life of Wireless Sensor Network [12]-This paper focuses on the problem that is encountered in most of the sensor network i.e. few nodes in the network dies earlier than the others. It focuses on how 
energy consumption can be minimized so that lesser energy is consumed and the network lifetime is increased.

The solution proposed uses a layered architecture. Based on the distance from base station the sensor nodes are distributed in layers, each layer is at $\mathrm{R}$ distance from the layer before it. Layer 1 is at $\mathrm{R}$ distance from the base station, Layer 2 is at $\mathrm{R}$ distance from layer 1 and so on. The use of layered architecture helps in sending data from lower to higher layer using minimum transmission energy. Transmission energy is directly proportional to minimum distance $\mathrm{R}$, so minimum energy is required to transmit data between layers.

A concept of Data Collection Range is also used in the paper which is defined at each layer. Data collection range depends on three factors:

- Battery Power of Wireless Sensor Node

- Transmission Range of Wireless Sensor

- Density of WSN per $\mathrm{m}^{2}$

All nodes in the data collection range $\mathrm{R}$ fuses their data at a maximum energy node and forwards the data to the next lower level. The simulation results show that the proposed model is better than PEAGSIS and LEACH.

\section{A Novel Clustering Algorithm for Energy} Efficiency in Wireless Sensor Networks (ANCAEE) [13] -The algorithm follows a simple concept of data transmission i.e. one hop between cluster members and the $\mathrm{CH}$ and multi hop from $\mathrm{CH}$ to base station. The algorithm proceeds in round, after $M$ rounds each node must have been elected as $\mathrm{CH}$ once; the value of $\mathrm{M}$ is calculated by dividing the total number of sensor nodes in the network by the total number of clusters. A variable is used to check weather a node can become a $\mathrm{CH}$ for the round or not, if the value of that variable for a node is even the node can be elected as $\mathrm{CH}$ if the value is odd it cannot be. In the cluster formation phase the $\mathrm{CH}$ broadcasts $A D V$ message using CSMA scheme, the nodes decide which $\mathrm{CH}$ to join and sends an acknowledgment massage back to the $\mathrm{CH}$. TDMA scheme is used to by cluster members to send data to $\mathrm{CH}$. In the steady state phase $\mathrm{CH}$ removes redundant data from the data it has received and then compresses the data. The compressed data is transmitted to the base station in a form of packet.

\section{Partitions and Sector Based Clustering Protocols. \\ Partition based LEACH algorithm ( $p$-LEACH)}

[14] - In this paper a partition based LEACH algorithm is proposed (p-LEACH) in which firstly the network is partitioned into sectors then a $\mathrm{CH}$ is elected in each sector using centralized calculations, the node having highest energy is elected as the head node.

The p-LEACH algorithm works in two steps: In the first step optimal number of CH's is calculated by the sink node and accordingly network is portioned into sectors. If the optimal number of CH's is $\mathrm{K}$ then network is divided into $\mathrm{K}$ sectors each having approx. same number of nodes, the distance between non cluster head and cluster head node is smaller than network radius and the deviation of distance from a nonhead node to head node is kept small to minimize energy consumption of the network. In the second step the highest energy node is selected as $\mathrm{CH}$ in each sector by the sink node then the information about elected $\mathrm{CH}$ is transmitted to whole network by the sink node.
In pLEACH algorithm energy is consumed in initialization phase when all nodes needs to send information to sink node or to receive a reply, after each $\mathrm{CH}$ election when sink node needs to broadcast information and in stable transmission phase. Simulation results have shown that LEACH and LEACH-C does not ensure consistent and uniform location of $\mathrm{CH}$ 's that results in shorter lifetime of network, pLEACH removes this problem and shows significant improvement both in terms of variance of residual energy and number of survival of nodes.

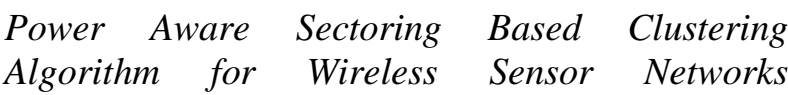
$(P A S C A L)$ [15] - In this paper the sensor field is hierarchically partitioned in levels of increasing signal strength and the levels are angularly partitioned into sectors of increasing angular measure. This protocol addresses some of the issues such as: Reducing the number of nodes that are used for transfer of data through leveling and sectoring, avoids flooding in the network, energy efficient path used to transfer data to base station and hence power aware., reliability of gateways increased and complexity of network is decreased. The protocol has four main phases:

Leveling: Leveling has two different approaches

Approach I: Base station transmits signals at various power levels, at the time of initial deployment signal of power 1 is transmitted by base station, all the nodes that receives this information sets their level as 1 . Next the signal power is increased for level 2. This process continues until signals corresponding to all the levels are sent by base station. The network is divided into levels corresponding to number of power levels send by the base station.

Approach II: Hop count technique is used to determine levels. Initially hop count of all nodes is set to infinity. The sink broadcast packets having hop count set to zero, nodes that receive this packet set their level as $l_{1}$ and increments the hop count field by 1 . The updated packets are broadcasted again and the levels of node updated accordingly.

Sectorization: The sensor field is divided into equiangular sectors in clockwise or anti clockwise direction after which BS broadcasts Signal broadcast packet(SBP) containing its own location information and Sector ID's

Clustering: Clustering uses a modified version of HEED algorithm. After election of initial $\mathrm{CH}$ non cluster head joins the cluster that requires minimum communication energy. The number of clusters is increased as we move farther from base station.

Routing Protocol: When any event occur the protocol works as follows: The packets are flooded, from the node where event occurred, by the cluster head to the other cluster heads in the direction of the Base Station. Every node that receives packets checks for two parameters namely the Level id and the Sector id from which the packet is propagated, the packet is accepted if it is from a higher level node and then the sector ID is checked, the packet is forwarded to other nodes if the packet is from the one-hop sector of higher level and if the packet is from the same level it is discarded. The packets received from the same sector IDs of the same level are also discarded. 
Sector based Multi-hop Clustering Protocol for Wireless Sensor Networks (SBMC) [16]-The SBMC protocol has the main goal of increasing the lifetime of the network. It uses three main concept viz. sectoring, leveling, multi hop clustering. The energy loss in discovering route for dissemination of data from $\mathrm{CH}$ to base station is minimized. In SMBC nodes are assigned unique id and the nodes are aware of their location. The algorithm has three main steps: in the first step that is partitioning clusters are formed by dividing the area into level and sectors, each cluster in the network is identified by level id and sector ID. The cluster is a curved area that is formed by intersection of circular levels and triangular section. It is further divided into two stages: level setup and sector setup stage.

In the second step $\mathrm{CH}$ is elected $\mathrm{AREQ}$ packet is broadcasted into the network by base station, nodes receiving this packet send their level ID; sector ID, node ID, energy level and location information to the base station. Based on this information, distance table for each cluster is calculated. $\mathrm{CH}$ for the initial round is also selected by taking energy, number of neighbors and how close to the center of the cluster into account. In the third step routing is done TDMA slots are created and distributed to all the members of the cluster. Each node can transmit during its slot, after assignment of TDMA slot data collection begins with the farthest node in the cluster. The farthest node sends data to nearest node, the nearest node adds its own data to the received data and transmit it furthers, the process continues until data of all member node reaches to $\mathrm{CH}$. Multi hop clustering is then used for data transmission to BS by CH's. The performance of SBMC is far better than the LEACH algorithm.

\section{COMPARISON OF THE}

\section{STRATEGIES}

In this section we evaluate the performance of various algorithms. In the first table the lifetime characteristics of the various algorithms are compared with LEACH.

TABLE 1: Performance evaluation of algorithms compared to LEACH

\begin{tabular}{|l|l|}
\hline Algorithm name & Performance \\
\hline ANCAEE & $\begin{array}{l}\text { Transmits twice the amount of data to } \\
\text { base station compared to LEACH, } \\
\text { lifetime of network increased } \\
\text { considerably }\end{array}$ \\
\hline $\begin{array}{l}\text { A new layered } \\
\text { architecture } \\
\text { approach }\end{array}$ & $\begin{array}{l}\text { Network performs 4 times better than } \\
\text { the LEACH algorithm. }\end{array}$ \\
\hline HCTE & $\begin{array}{l}\text { Network lifetime is increased by 35\% } \\
\text { compared to LEACH }\end{array}$ \\
\hline pLEACH & $\begin{array}{l}\text { Network lifetime increased by 20\% } \\
\text { compared to LEACH }\end{array}$ \\
\hline
\end{tabular}

\begin{tabular}{|l|l|}
\hline EECS & $\begin{array}{l}\text { Improvement of 35\% in network } \\
\text { lifetime compared to LEACH }\end{array}$ \\
\hline TL-LEACH & $\begin{array}{l}\text { 30\% improvement in network lifetime } \\
\text { compared to LEACH }\end{array}$ \\
\hline SBMC & $\begin{array}{l}\text { Network lifetime increased by 13\% } \\
\text { compared to LEACH }\end{array}$ \\
\hline
\end{tabular}

In the second table the algorithms are compared based on first node dead time (FND) and last node dead time (LND) for a given number of nodes.

TABLE 2: Comparison on the basis FND and LND for various algorithms

\begin{tabular}{|l|l|l|l|}
\hline Algorithm & FND & LND & $\begin{array}{l}\text { No. of } \\
\text { nodes }\end{array}$ \\
\hline LEACH & $\begin{array}{l}\text { After 135 } \\
\text { round }\end{array}$ & $\begin{array}{l}\text { After 640 } \\
\text { round }\end{array}$ & 100 \\
\hline TL-LEACH & $\begin{array}{l}\text { After 148 } \\
\text { rounds }\end{array}$ & $\begin{array}{l}\text { After 710 } \\
\text { rounds }\end{array}$ & 100 \\
\hline ACNEE & $\begin{array}{l}\text { After 185 } \\
\text { round }\end{array}$ & $\begin{array}{l}\text { After } 800 \\
\text { rounds }\end{array}$ & 100 \\
\hline SBMC & $\begin{array}{l}\text { After 820 } \\
\text { rounds }\end{array}$ & $\begin{array}{l}\text { After 1250 } \\
\text { rounds }\end{array}$ \\
\hline HCTE & $\begin{array}{l}8 \% \\
\text { optimization } \\
\text { LEACH }\end{array}$ & $\begin{array}{l}\text { 24\% } \\
\text { optimization } \\
\text { compared to } \\
\text { LEACH }\end{array}$ & 200 \\
\hline
\end{tabular}

\section{CONCLUSION}

This paper has tried to discuss and summarize most of the pioneer and the recent approaches for advanced clustering of wireless sensor nodes. Clearly the approaches have undergone an evolution to reach to the state of the art, still a long way to go for enhancement of the network life time. The power of the node has to be used in more secure and efficient ways. The hierarchical architecture based clustering networks have been experimentally proved the best for a predefined WSN, but the major drawbacks of these networks is early exhausting of the nodes that are in the lower level of hierarchy and take part in almost all the communications. A brief comparison of the approaches has been summarized in the tables.

This paper has attempted to draw attention of the WSN research community towards pros of the architecture based networks. Their performances can be drastically enhanced by adapting the approaches of the dynamic cluster head selections. 


\section{REFERENCES}

[1] F. Akyildiz, W.Su, Y. Sankarasubramaniam, and E.Cyirci, Wireless sensor networks: a survey, Computer Networks, pp. 393-422, March 2002.

[2] D.J. Baker, A. Ephremides, J.A. Flynn, The design and simulation of a mobile radio network with distributed control, IEEE Journal on Selected Areas in Communications (1984) 226-237.

[3] S. Banerjee, S. Khuller, A clustering scheme for hierarchical control in multi-hop wireless networks, in: Proceedings of 20th Joint Conference of the IEEE Computer and Communications Societies (INFOCOM' 01), Anchorage, AK, April 2001

[4] W.B. Heinzelman, A.P. Chandrakasan, H. Balakrishnan, "Application specific protocol architecture for wireless micro sensor networks," IEEE Transactions on Wireless Networking, 1(4), 2002, pp.660-670

[5] .O. Younis, S. Fahmy, HEED: A Hybrid, EnergyEfficient, Distributed clustering approach for Ad Hoc sensor networks, IEEE Transactions on Mobile Computing 3 (4) (2004) 366-379.

[6] S. Bandyopadhyay, E. Coyle, An energy efficient hierarchical clustering algorithm for wireless sensor networks, in: Proceedings of the 22nd Annual Joint Conference of the IEEE Computer and Communications Societies (INFOCOM 2003), San Francisco, California, April 2003

[7] LI-QING GUO, YI XIE*, CHEN-HUI YANG, ZHENGWEI JING. Improvement on leach by combining adaptive cluster head election and two-hop transmission. In the Proceedings of the Ninth International Conference on Machine Learning and Cybernetics, Qingdao, 11-14 July 2010.

[8] V. Loscri, G. Morabito and S. Marano, "A Two-Level Hierarchy for Low-Energy Adaptive Clustering Hierarchy," 62nd IEEE Vehicular Technology Conference, Vol. 3, No. 2, 2005, pp. 1809-1813.
[9] NasrinAzizi, JaberKarimpour, FaridSeifi . HCTE: Hierarchical Clustering based routing algorithm with applying the Two cluster heads in each cluster for Energy balancing in WSN, in International Journal of Computer Science Issues, Vol. 9, Issue 1, No 2, January 2012,57 61

[10] P. Ding, J. Holliday, A. Celik, Distributed energy efficient hierarchicalclustering for wireless sensor networks, in: Proceedings of the IEEE International Conference on Distributed Computing in Sensor Systems (DCOSS'05), Marina Del Rey, CA, June 2005.

[11] Y. Mao, L. Chengfa, C. Guihai, and J. Wu "EECS: An energy efficient clustering scheme in wireless sensor networks". In Proceedings IPCCC, IEEE 24th International, 535-540.

[12] Babu Ram, Narottam Chand, Prateek Gupta, Siddhartha Chauhan, A New Approach Layered Architecture based Clustering for Prolong Life of Wireless Sensor Network (WSN) in the International Journal of Computer Applications (0975 - 8887) Volume 15- No.1, February 2011

[13] Ademola P. Abidoye, Nureni A. Azeez Ademola O. Adesina1,Kehinde K. Agbele1, ANCAEE: A Novel Clustering Algorithm for Energy Efficiency in Wireless Sensor Networks, In Wireless Sensor Network, 2011, 3 307-312

[14] Haosong Gou, YounghwanYoo, Hongqing Zeng. A partition based LEACH algorithm, in IEEE Ninth International Conference on Computer and Information Technology. 2009.40-45.

[15] Md.Aquil Mirza, Rama Murthy Garimella, PASCAL: Power Aware Sectoring Based Clustering Algorithm forWireless Sensor Networks.

[16] Bore Gowda S B, Puttamadappa C, Mruthyunjaya H S, Babu N V. Sector based Multi-hop Clustering Protocol for Wireless Sensor Networks in International Journal of Computer Applications (0975 - 8887) Volume 43No.13, April 2012. 